\title{
Color Characterization Comparison for Machine Vision-Based Fruit Recognition
}

\author{
Farid García-Lamont ${ }^{1(\bowtie)}$, Jair Cervantes ${ }^{1}$, Sergio Ruiz ${ }^{1}$, \\ and Asdrúbal López-Chau ${ }^{2}$ \\ ${ }^{1}$ Centro Universitario UAEM Texcoco, \\ Universidad Autónoma del Estado de México, Av. Jardín Zumpango s/n, \\ Fraccionamiento El Tejocote, 56259 Texcoco-Estado de México, Mexico \\ fgarcial@uaemex.mx, \{chazarral7, jsergioruizc\}@gmail.com \\ ${ }^{2}$ Centro Universitario UAEM Zumpango, \\ Universidad Autónoma del Estado de México, \\ Camino viejo a Jilotzingo continuación Calle Rayón, \\ 55600 Zumpango-Estado de México, Mexico \\ alchau@uaemex.mx
}

\begin{abstract}
In this paper we present a comparison between three color characterizations methods applied for fruit recognition, two of them are selected from two related works and the third is the authors' proposal; in the three works, color is represented in the RGB space. The related works characterize the colors considering their intensity data; but employing the intensity data of colors in the RGB space may lead to obtain imprecise models of colors, because, in this space, despite two colors with the same chromaticity if they have different intensities then they represent different colors. Hence, we introduce a method to characterize the color of objects by extracting the chromaticity of colors; so, the intensity of colors does not influence significantly the color extraction. The color characterizations of these two methods and our proposal are implemented and tested to extract the color features of different fruit classes. The color features are concatenated with the shape characteristics, obtained using Fourier descriptors, $\mathrm{Hu}$ moments and four basic geometric features, to form a feature vector. A feed-forward neural network is employed as classifier; the performance of each method is evaluated using an image database with 12 fruit classes.
\end{abstract}

Keywords: Color characterization · Fruit classification · RGB images

\section{Introduction}

The automated recognition of different classes of fruits using artificial vision, has been a few studied. Related works have focus mainly on to evaluate the ripeness or quality of fruits [1-4] or to recognize varieties of the same fruit class [5-8]; that is, they do not recognize different classes of fruits. Reviewing the state of the art, we have found the proposal presented in references [9-11], where fruit recognition is performed by extracting color, shape and texture features, but texture is not always employed. Color is an important feature for fruit recognition, especially when the shapes of fruits are very similar. For instance, the shapes of tomato and orange are almost the same; 


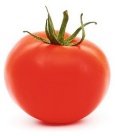

(a)

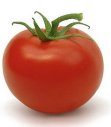

(b)

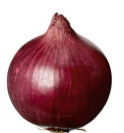

(c)

Fig. 1. Tomatoes (a) and (b) with the same chromaticity but different intensity, onion (c) with different chromaticity but with the same intensity as the tomato (b)

however, the color feature becomes a discriminative data to classify the fruits successfully.

About color characterization, in reference [9] the color is extracted by discretizing each color channel in 4 intensity levels, then a histogram is computed where the occurrences of the $4 \times 4 \times 4=64$ possible colors are counted. In [10] the color is modeled by computing the mean of each color channel; these values are concatenated to form the feature vector of color. Betul et al. [11] characterize the color with fuzzy logic, where the inference system models the colors employing the intensity values of the color channels. In these works the feature vector of the fruit is obtained by concatenating the color and shape features; in [9] texture features are also included.

The drawback with the color extraction of these three approaches is that they depend on the intensity of colors. For instance, tomatoes (a) and (b) shown in Fig. 1 have the same red hue, but their intensities are different. Humans can associate this red hue to tomatoes, independently of the intensity; but in the RGB space, because of the intensity difference, they represent different colors. On the other hand, the onion (c) can be recognized due to its characteristic red hue, despite the intensity of the onion is similar to tomato (b).

The contribution of this paper consists on to show that fruit recognition can be more precise if color chromaticity is employed to model the color of fruits. Fruits have a characteristic color; due to the fruits can have different levels of ripeness, the intensity is different between fruits of the same class but the chromaticity changes just a little. With our approach it is possible to maintain the data of chromaticity despite intensity changes.

The rest of the paper is organized as follows: in Sect. 2 the methods for color and shape characterization are introduced, where our proposal is presented. Experiments with a fruit image database are performed in Sect. 3. In Sect. 4 the results are discussed, conclusions and future work in Sect. 5 close the paper.

\section{Feature Extraction}

In this section we present the methods employed to extract color and shape features of fruits. Figure 2 shows the steps we propose to characterize and recognize fruits.

First, the image of the fruit is acquired in the RGB space and then the image is mapped to the HSV space. It is important to remark that the background of the acquired images must be white, and in the scene there is just one kind of fruit. By using data about the saturation of colors, the fruit is segmented from the background. After 


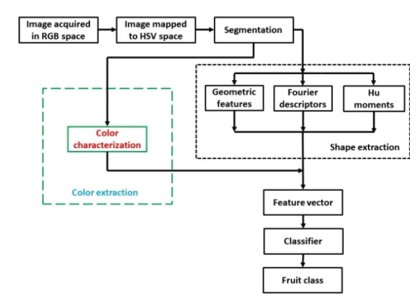

Fig. 2. Flow chart of the proposed approach

segmentation, the color and shape features are extracted. In Sect. 2.1 we explain our proposal for color characterization; in Sect. 2.2 the Fourier descriptors, $\mathrm{Hu}$ moments and basic geometric feature extraction methods are explained for shape characterization. The color and shape characteristics are concatenated to form a feature vector, which is feeded to a classifier and finally we obtain the class of the fruit.

\subsection{Color Characterization}

Before we introduce our proposal, it is convenient to give a short explanation of color representation in the RGB and HSV spaces. The RGB (Red, Green, Blue) space is based in a Cartesian coordinate system where colors are points defined by vectors that extend from the origin, where black is located in the origin and white in the opposite corner to the origin, see Fig. 3.

The color of a pixel $p$ is written as a linear combination of the basis vectors red, green and blue, that is:

$$
\phi_{p}=r_{p} \hat{i}+g_{p} \hat{j}+b_{p} \hat{k}
$$

Where $r_{p}, g_{p}$ and $b_{p}$ are the red, green and blue components, respectively. The orientation and magnitude of a color vector defines the chromaticity and the intensity of the color, respectively [12].

The HSV space is cone shaped, see Fig. 4, the representation of the color of a pixel $p$ in the HSV space is written as [13]:

$$
\varphi_{p}=\left[h_{p}, s_{p}, v_{p}\right]
$$

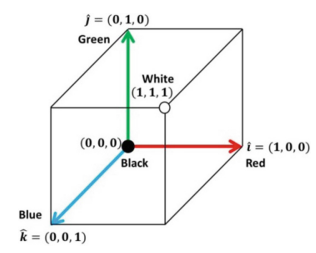

Fig. 3. RGB color space 


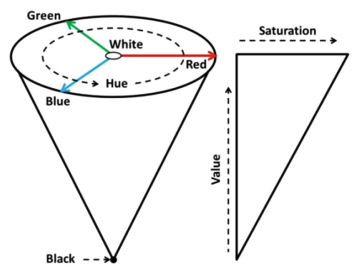

Fig. 4. HSV color space

Where $h_{p}, s_{p}$ and $v_{p}$ are the hue, saturation and value components, respectively. The hue is the chromaticity, saturation is the distance to the glow axis of black-white, and value is the intensity. The real ranges of the hue, saturation and value components are $[0,2 \pi],[0,1]$ and $[0,255]$, respectively.

As mentioned previously, we assume that in the image there is one kind of fruit and the background is white. The acquired image is mapped to the HSV space, the pixel's intensity is set to zero if its saturation is lower than a specific threshold.

As shown in Fig. 4, the white color is located in the axis of the cone; that is, white is a low saturated color. Thus, the color of the pixels with low saturation is white; therefore they are part of the image background.

The segmentation process is not performed in the RGB space because the computational load may be huge; to establish if a color vector represents white color, its orientation must be computed and compared to a specific reference value. By comparing the white color by its saturation, the computational load lies just in the image mapping from RGB space to HSV space; however, the computational load is low. The color extraction we propose consists on the following steps:

1. Let $\left\{\phi_{1}, \ldots, \phi_{m}\right\} \subset \mathbf{R}^{3}$ be the set of color vectors of the image's pixels, represented in the RGB space. The color vectors are mapped to the HSV space and we obtain the set of vector $\left\{\varphi_{1}, \ldots, \varphi_{m}\right\} \subset \mathbf{R}^{3}$. The segmentation is performed by comparing the saturation of the colors. If the saturation is lower than a threshold, then the color vector of the corresponding pixel in the RGB space is set to zero. In other words:

$$
\phi_{p}^{*}=\left\{\begin{array}{cc}
\phi_{p}, & s_{p} \geq t h \\
\overrightarrow{0} & s_{p}<t h
\end{array}\right.
$$

Where $t h$ is the threshold value and $\overrightarrow{0}=[0,0,0]$. In this step the fruit is segmented from the background. In this study $t h=0.2$. Figure 5 shows the images of two fruits segmented from the background using this segmentation approach.

2. The set $S$ is built as follows:

$$
S=\left\{\phi_{p}^{*} \mid \phi_{p}^{*} \neq \overrightarrow{0}\right\}
$$




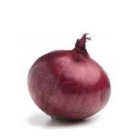

(a)

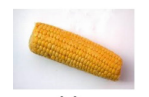

(c)

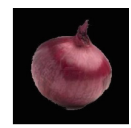

(b)

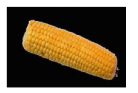

(d)

Fig. 5. Examples of segmented images: (a) and (c) input images, (b) and (d) images obtained after segmentation

3. The vectors of set $S$ are sum, i.e.,

$$
R=\sum_{\phi_{p}^{*} \in S} \phi_{p}^{*}
$$

4. The vector $R$ includes data of all the colors of the object, its orientation is the same or almost the same of the color vectors of the dominant color. It also includes data of the other colors of the fruit. The magnitude of vector $R$ is very large; however, what we are interested on is the orientation of vector $R$ because it provides data about the chromaticity of the color obtained from the fruit. Hence, the vector is normalized with:

$$
u_{R}=\frac{R}{\|R\|}=\left[r_{u}, g_{u}, b_{u}\right]
$$

Thus, the vector $u_{R}$ characterizes the color of the fruit; the direction cosines of this vector are $\cos \alpha_{R}=r_{u} /\left\|u_{R}\right\|, \cos \beta_{R}=g_{u} /\left\|u_{R}\right\|$ and $\cos \theta_{R}=b_{u} /\left\|u_{R}\right\|$. But $\left\|u_{R}\right\|=1$, therefore, the components of the vector $u_{R}$ are the cosines of the angles between the vector and the basis vectors. Hence, the orientation of $R$ is implicit in $u_{R}$. Therefore, the vector $u_{R}$ characterizes the fruit's color by its chromaticity and the effect of intensity is reduced.

\subsection{Shape Features}

The shape features are characterized using Fourier descriptors, $\mathrm{Hu}$ moments and geometrical features. In order to compute the shape features we build the set $O=\left\{\left(x_{0}, y_{0}\right), \ldots,\left(x_{n-1}, y_{n-1}\right)\right\}$, whose elements are the coordinates of the pixels of the segmented area. The shape features are computed as follows.

Fourier Descriptors: The Fourier descriptors compute the set of points of the object's contour as a sequence of complex numbers. With this data is built a periodic unidimensional function $f$ that describes the object's contour as a sequence of complex numbers; the coefficients of the Fourier transform of function $R$ characterizes the contour of an object in the frequency domain [14]. Let $C \subset O$ be the set whose 
elements are the coordinates of the contour's pixels of the segmented area. The coordinates in $C=\left\{\left(x_{0}, y_{0}\right), \ldots,\left(x_{M-1}, y_{M-1}\right)\right\}$ are indexed according to the sequence they are located in the contour at a specific direction and starting at an arbitrary point. Thus, being $i=\sqrt{-1}$ and $\mathbf{C}$ the set of complex numbers, let $f: C \rightarrow \mathbf{C}$ be a function defined as follows:

$$
f\left(\left(x_{k}, y_{k}\right)\right)=x_{k}+i y_{k}
$$

The Fourier transform of function $\mathrm{f}$ is computed with:

$$
F(u)=\frac{1}{M} \sum_{k=0}^{M-1} f\left(\left(x_{k}, y_{k}\right)\right) \exp \left(\frac{-i 2 \pi u k}{M}\right)
$$

The complex coefficients $F(u)$ are known as the contour's Fourier descriptors. In this work we employ eight coefficients, that is, $F(u)$ is computed for $u=0,1, \ldots, 7$.

Hu moments: $\mathrm{Hu}$ moments compute the features of object's shape, considering the shape as a distribution of coordinates of $2 \mathrm{D}$ points $[15,16]$. It is based in the central moments of the coordinates' distribution, which express characteristic parameters respect to a centroid point. Thus, let $O$ be the set defined previously, the Hu moments are computed with:

$$
\begin{aligned}
H_{1}= & \eta_{20}+\eta_{02} \\
H_{2}= & \left(\eta_{20}-\eta_{02}\right)^{2}+4 \eta_{1}^{2} \\
H_{3}= & \left(\eta_{30}-3 \eta_{12}\right)^{2}+\left(3 \eta_{21}-\eta_{03}\right)^{2} \\
H_{3}= & \left(\eta_{30}-3 \eta_{12}\right)^{2}+\left(3 \eta_{21}-\eta_{03}\right)^{2} \\
H_{4}= & \left(\eta_{30}+\eta_{12}\right)^{2}+\left(\eta_{21}+\eta_{03}\right)^{2} \\
H_{5}= & \left(\eta_{30}-3 \eta_{12}\right)\left(\eta_{30}+\eta_{12}\right)\left[\left(\eta_{30}+\eta_{12}\right)^{2}-3\left(\eta_{21}+\eta_{03}\right)^{2}\right] \\
& +\left(3 \eta_{21}-\eta_{03}\right)\left(\eta_{21}+\eta_{03}\right)\left[3\left(\eta_{30}+\eta_{12}\right)^{2}-\left(\eta_{21}+\eta_{03}\right)^{2}\right] \\
H_{6}= & \left(\eta_{20}-\eta_{02}\right)\left[\left(\eta_{30}+\eta_{12}\right)^{2}-\left(\eta_{21}+\eta_{03}\right)^{2}\right]+4 \eta_{11}\left(\eta_{30}+\eta_{12}\right)\left(\eta_{21}+\eta_{03}\right) \\
H_{7}= & \left(3 \eta_{21}-\eta_{03}\right)\left(\eta_{30}+\eta_{12}\right)\left[\left(\eta_{30}+\eta_{12}\right)^{2}-3\left(\eta_{21}+\eta_{03}\right)^{2}\right] \\
& -\left(\eta_{30}-3 \eta_{12}\right)\left(\eta_{21}+\eta_{03}\right)\left[3\left(\eta_{30}+\eta_{12}\right)^{2}-\left(\eta_{21}+\eta_{03}\right)^{2}\right]
\end{aligned}
$$

The centralized and normalized moments $\eta_{p q}$ are obtained with:

$$
\eta_{p q}=\frac{\mu_{p q}}{\mu_{00}^{c+1}}
$$


Where $c=(p+q) / 2$. The centralized moments are computed with:

$$
\mu_{p q}(O)=\sum_{(x, y) \in O}(x-\bar{x})^{p}(y-\bar{y})^{q}
$$

Where $\bar{x}$ and $\bar{y}$ are the mean values of the $x$ and $y$ values of coordinates of set $O$.

Basic geometric features: In this work we extract four features: eccentricity, solidity, compaction and roundness. Eccentricity is the ratio of the distance between the foci of the ellipse and its major axis length. Solidity is the proportion of the pixels in the object that are also in the convex hull.

Compaction is the relation between the area of an object and its perimeter:

$$
C p(O)=\frac{n}{P(O)}
$$

Roundness is computed with:

$$
R(O)=\frac{4 \pi n}{P^{2}(O)}
$$

Where $P(O)$ is the perimeter of object $O$. All these features are concatenated to form the feature vector whose dimension is 22 . It is important to mention that all the shape features extracted are invariant to rotation, translation and scale.

\section{Experiments and Results}

The experimental set up consists on to classify twelve classes of fruits: red onion, green chili, corn, melon, orange, potato, pear, cucumber, pineapple, banana, tomato and carrot; hand labeled as classes 1, 2,..,12, respectively. Figure 6 shows images of the
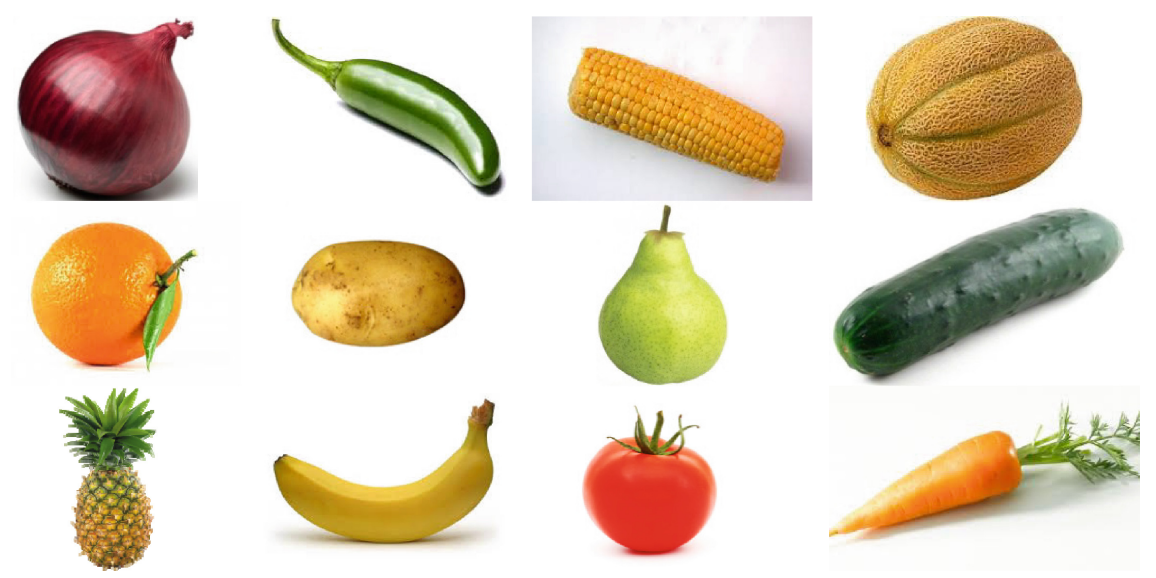

Fig. 6. Samples of the twelve fruit classes employed for experiments 
fruit classes recognized in this study. In order to appreciate easily the influence of the color characterizations, we select fruits with similar appearance. For instance, red onion and tomato, green chili and cucumber, melon and potato, banana and corn.

The image database employed has 720 images, 60 per fruit class; half of the image database is employed for training and the other half of the image database is used for testing. The image database was built by downloading images from internet and by photos acquired using a digital camera. The images were acquired such that in the scene there is only one fruit and the color of background is white. The fruit is acquired in the same position and angle in order to ease the shape feature extraction due to what we are interested on is the color characterization. The size of the images varies from $160 \times 159$ to $1000 \times 965$ pixels.

A feed-forward neural network (NN) is employed to recognize the fruits. The NN has three layers; one input layer, one hidden layer and one output layer. The first and second layers have 25 neurons and the third layer has 12 neurons. The activation function for the first and second layers is hyperbolic tangent sigmoid, while the activation function of the third layer is log-sigmoid. Table 1 shows the recognition rates obtained with our proposal and the methods presented in references $[9,10]$.

The highest recognition rate is obtained with our proposal; it is important to know between which fruit classes there are misclassifications, so as to analyze which color characterization works better. Thus, we present the confusion matrixes obtained with each of the three color characterizations implemented in this study. Table 2 shows the confusion matrix using the proposal of Zhang et al. [9].

The fruits best classified are red onion, melon, orange, potato, cucumber and tomato; the fruit worst classified is pineapple.

Table 1. Recognition rates obtained using the approaches presented in references $[9,10]$, and by the authors

\begin{tabular}{l|l|l|l}
\hline \multirow{2}{*}{ Fruit class } & \multicolumn{3}{l}{ Recognition rate } \\
\cline { 2 - 4 } & Zhang et al. [9] & Chaw and Hadi [10] & Authors' proposal \\
\hline Red onion & 93.34 & 96.67 & 100 \\
\hline Green chili & 86.67 & 83.33 & 86.67 \\
\hline Corn & 86.67 & 90 & 93.34 \\
\hline Melon & 93.34 & 90 & 93.34 \\
\hline Orange & 93.34 & 93.34 & 93.34 \\
\hline Potato & 93.34 & 80.00 & 93.34 \\
\hline Cucumber & 86.67 & 90.00 & 90.00 \\
\hline Pear & 93.34 & 83.34 & 96.67 \\
\hline Pineapple & 80.00 & 73.34 & 86.67 \\
\hline Banana & 90.00 & 96.67 & 96.67 \\
\hline Tomato & 93.34 & 93.34 & 100 \\
\hline Carrot & 90.00 & 86.67 & 96.67 \\
\hline Average & 90.01 & 88.06 & 93.89 \\
\hline
\end{tabular}


Table 2. Confusion matrix obtained employing the proposal of reference [9]

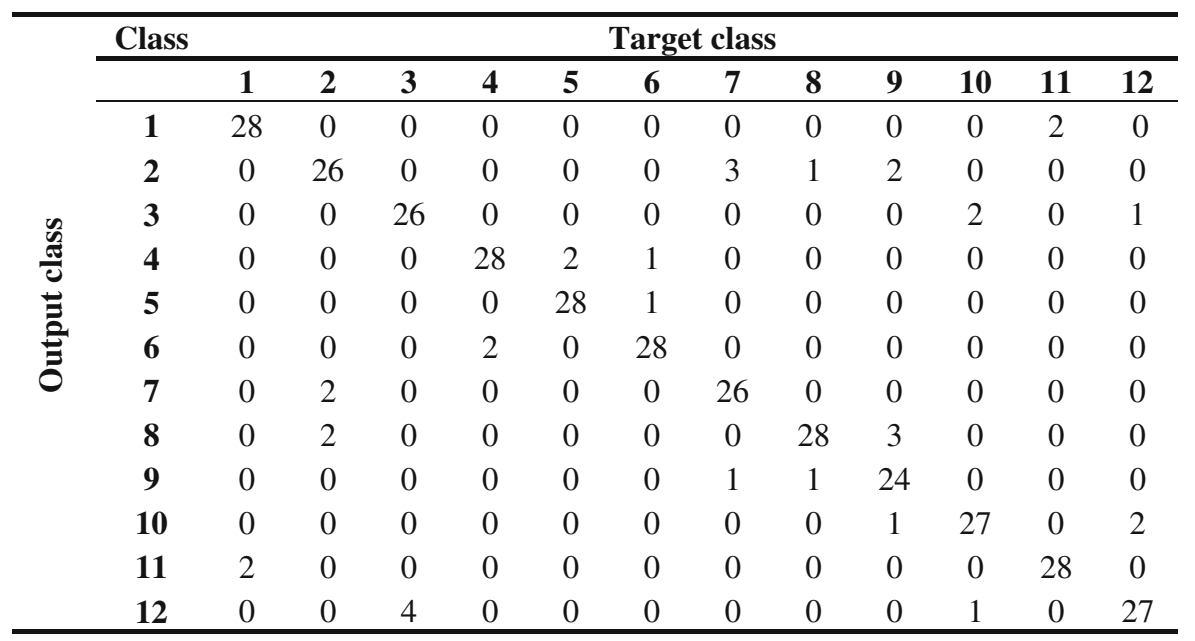

Table 3 shows the confusion matrix employing the color characterization used by Chaw and Hadi [10]. The fruits best classified are red onion and banana; the worst classified fruit is pineapple.

Table 4 shows the confusion matrix using the color characterization proposed by the authors.

The fruits best classified are red onion and tomato, the fruits worst classified are green chili and pineapple. In the following Sect. 4 we analyze and discuss the results shown in the current section.

Table 3. Confusion matrix obtained using the approach of reference [10]

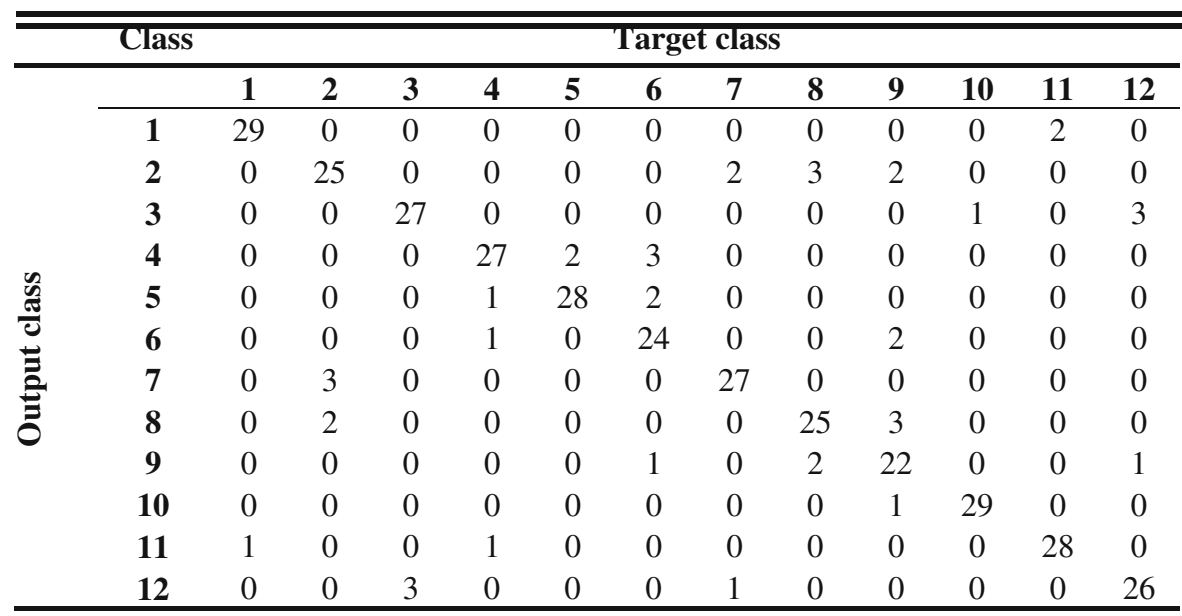


Table 4. Confusion matrix obtained using the authors' proposal

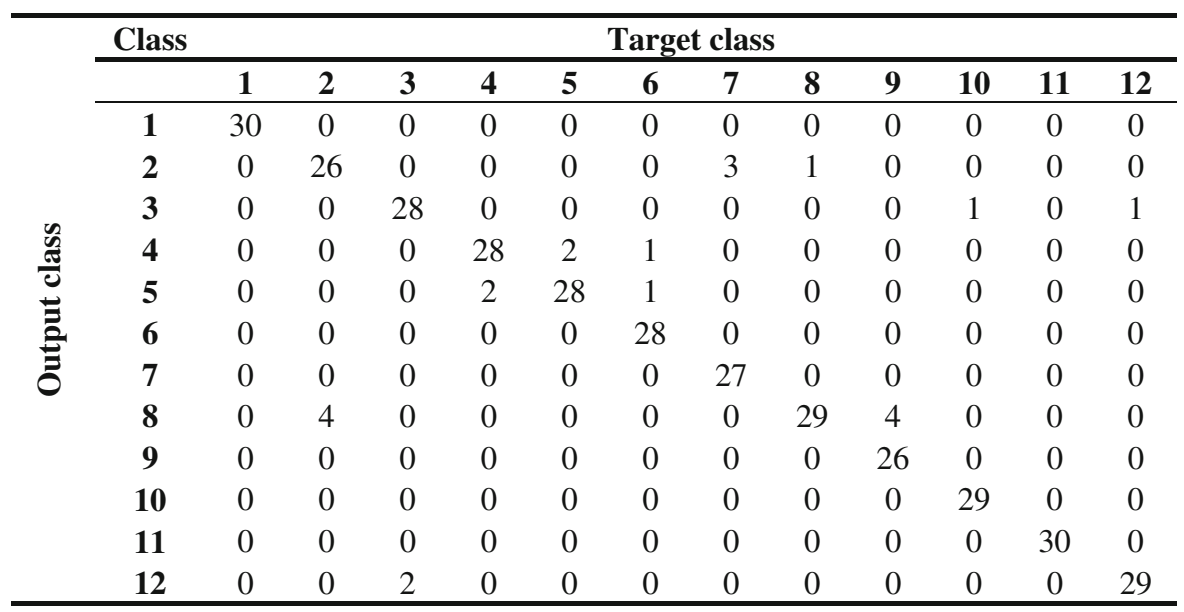

\section{Discussion}

According to the results of Table 1 the highest recognition rate is $93.89 \%$, which is obtained with our proposal; the second and third recognition rates are $90.01 \%$ and $88.06 \%$, obtained with the methods presented in references $[9,10]$, respectively. The fruit worst classified by the three methods is pineapple. The cause may be due to the shape of pineapples is more complex than the other fruits, thus, it may be necessary to extract more shape features. Red onion is the best classified fruit by the three methods.

As stated in Sect. 1, color and shape features are extracted for fruit recognition. Because of the shape features, some fruits are misclassified, not by the color characterization; for instance pineapples and carrots. Hence, it is necessary to analyze the confusion matrixes built with the data obtained with the three methods.

From the results of Tables 2, 3 and 4, most of the red onions are recognized correctly, the misclassified onions are recognized as tomatoes; except with our proposal, where all the onions are recognized successfully.

The misclassified chilies are recognized as pears or cucumbers. With our method, all the chili misclassifications are assigned to cucumbers because the shape and chromaticity of both fruits are akin. With the other methods, the chilies misclassified as pears occur because the intensity of these chilies is as large as the pears. In other words, by employ intensity data of color, some chilies have the same or almost the same intensity of pears color; thus, these chilies are misclassified as pear.

With the three methods, the corn misclassifications are assigned to the carrot class. A plausible explanation is that, besides more shape features are needed, the color combination of carrots is a yellow-like hue; due to carrots usually have green and orange hues. 
Misclassifications of melon class are with potato and orange. The color between potato and orange may be similar; the color of melons tends to the color of oranges, depending on the ripeness of the fruit.

Similarly to the melon class case, the orange class is misclassified melon. The color of the misclassified oranges is akin to the color of melon. The misclassification happens not only because of the color feature, but also the shape of both fruits is almost the same. To overcome the misclassification it may be necessary to extract more shape features or to obtain texture features.

Potato class is misclassified as melon and/or orange because the color of the misclassified potatoes is similar to some melons and oranges, not to mention that the shape is alike, to some extent.

With the methods of references [9, 10], some pears are misclassified as green chili and pineapple. As explained before, the color extracted from these misclassified pears has almost the same intensity of the chili color. Misclassifications with pineapples occur because pineapples have some parts with green color.

Cucumber is misclassified with green chili and pineapple by the methods of $[9,10]$, because the color and shape of both chili and cucumber are resembled; while cucumber is misclassified as pineapple due to pineapples have green hues. With our method, only one cucumber is classified as green chili.

Pineapple is classified as cucumber and banana; despite the complex shape of pineapple, due to the green and yellow hues and brightness of these colors, the pineapples are misclassified as cucumber and banana, respectively.

Banana is classified as corn and carrot, in this case the shape is not characterized correctly, so, the color of the fruit is the discriminant data for classification. In this case, yellow is the characteristic color of both banana and corn; thus, corn is misclassified as banana.

Tomato is misclassified as red onion; the brightness of those misclassified tomatoes is as low as the red onions, thus, they are classified as if they were the same fruit. Note that with our proposal all the tomatoes are classified successfully.

Carrot is classified as corn and banana, the color of carrots is a combination between orange and green, so, the resulting color is a color with yellow hue, to some extent. Therefore, carrot is misclassified as corn or banana.

It is important to remark that the dimension of the color feature vectors computed using the proposal of Zhang et al. [9] and ours is 64 and 3, respectively, The color features are concatenated with the 19 shape characteristics extracted in this work; thus, the dimension of the feature vector using the approach of reference [9] and ours is 83 and 22, respectively. Although the dimensionality of the feature vector can be performed using principal component analysis, computing the color feature vector as proposed in [9] can be a huge computational load.

The dimension of the color feature vector obtained with our proposal the approach presented in [10] is the same. But, as mentioned before, our proposal works better for fruit recognition because we employ the chromaticity data of fruits. While in [10] include the intensity data of colors; but in the RGB space despite the colors with the same chromaticity and with different intensities are classified as different colors. 


\section{Conclusions and Future Work}

In this work we have introduced an approach to characterize color by its chromaticity in the RGB space, applied for fruit recognition. The color features extracted with our proposal are concatenated with the shape features extracted using Fourier descriptors, $\mathrm{Hu}$ moments and basic geometric features to form a feature vector that characterizes the fruits. Our approach is compared with two methods of color characterization for fruit recognition of related works, which employ intensity data of colors. The three methods are tested with an image database containing 720 fruit images of 12 fruit classes. According to results, our proposal obtained the highest recognition rate, $93.89 \%$.

The current approach is planned to be applied on supermarkets, to help the cashiers to identify the different fruit classes; fruits do not have bar codes print on them that eases the automated classification and for cashiers it is difficult to memorize all the fruit codes. Hence, the future work is to strength the performance of this proposal by improving or adding the following points. Include intensity data of colors as a feature separated from the chromaticity. The HSV color space can be employed for this purpose because, in this space, the intensity is decoupled from the chromaticity.

To improve the accuracy by collecting more fruit images per class, acquired at different angles, positions and sizes. Increase the amount of fruit classes to recognize. Include more shape features, such as, increase the number of Fourier descriptors and/or basic geometric features. In this work we do not extract texture features; this is an important characteristic $[17,18]$ of fruits that will be employed in future studies.

\section{References}

1. Bhatt, A., Pant, D.: Automatic apple grading model development based on back propagation neural network and machine vision, and its performance evaluation. AI Soc. 30(1), 45-56 (2015)

2. Zhang, B., Huang, W., Li, W., Zhao, J., Fan, S., Wu, J., Liu, C.: Principles, developments and applications of computer vision for external quality inspection of fruits and vegetables: a review. Food Res. Int. 62, 326-343 (2014)

3. Sekhar, N.C., Tudu, B., Koley, C.: A machine vision-based maturity prediction system for sorting of harvested mangoes. IEEE Trans. Instrum. Meas. 63(7), 1722-1730 (2014)

4. Rodríguez-Pulido, F.J., Gordillo, B., González-Miret, M.L., Heredia, F.J.: Analysis of food appearance properties by computer vision applying ellipsoids to colour data. Comput. Electron. Agric. 99, 108-115 (2013)

5. van Henten, E.J., Hemming, J., Van Tuijl, B.A.J., Kornet, J.G., Meuleman, J., Bontsema, J., Van Os, E.A.: An autonomous robot for harvesting cucumbers in greenhouses. Auton. Robot. 13(3), 241-258 (2002)

6. Manickavasagan, A., Al-Mezeini, N., Al-Shekaili, H.: RGB color imaging technique for grading of dates. Sci. Hortic. 175, 87-94 (2014)

7. Chen, X., Yang, S.: A practical solution for ripe tomato recognition and localization. J. Real-Time Image Process. 8(1), 35-51 (2013)

8. Gatica, G., Best, S., Ceroni, J., Lefranc, G.: Olive fruits recognition using neural networks. Proc. Comput. Sci. 17, 412-419 (2013) 
9. Zhang, Y., Wang, S., Ji, G., Phillips, P.: Fruit classification using computer vision and feedforward neural network. J. Food Eng. 143, 167-177 (2014)

10. Chaw, S.W., Hadi, M.S.: A new method for fruits recognition system. In: International Conference on Electrical Engineering and Information, pp. 130-134 (2009)

11. Bostanci, B., Hagras, H., Dooley, J.: A neuro fuzzy embedded agent approach towards the development of an intelligent refrigerator. In: IEEE International Conference on Fuzzy Systems, pp. 1-8 (2013)

12. Gonzalez, R.C., Woods, R.E.: Digital Image Processing, 2nd edn. Prentice Hall, Upper Saddle River (2002)

13. Rotaru, C., Graf, T., Zhang, J.: Color image segmentation in HSI space for automotive applications. J. Real-Time Image Process. 3(4), 311-322 (2008)

14. Zahn, C., Roskies, R.: Fourier descriptors for plane closed curves. IEEE Trans. Comput. C-21(3), 269-284 (1972)

15. Hu, M.K.: Visual pattern recognition by moment invariants. IRE Trans. Inf. Theory 8(2), 179-187 (1962)

16. Wang, X., Huang, D.S., Du, J.X., Xu, H., Heutte, L.: Classification of plant leaf images with complicated background. Appl. Math. Comput. 205(2), 916-926 (2008)

17. Yuan, J.H., Huang, D.S., Zhu, H.D., Gan, Y.: Completed hybrid local binary pattern for texture classification. In: International Conference on Neural Networks, pp. 2050-2057 (2014)

18. Zhao, Y., Huang, D.S., Jia, W.: Completed local binary count for rotation invariant texture classification. IEEE Trans. Image Process. 21(10), 4492-4497 (2012) 